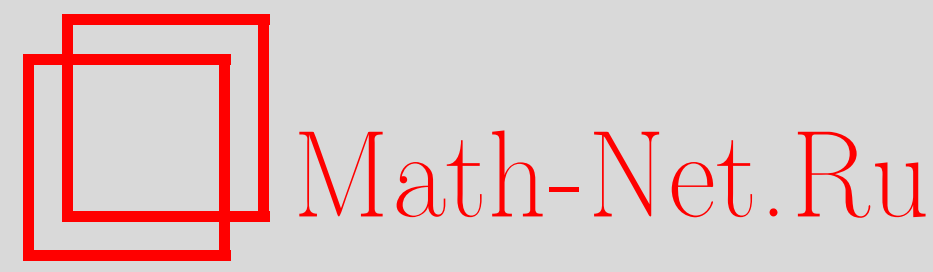

М. В. Зайцев, С. П. Мищенко, Критерий полиномиальности роста многообразий супералгебр Ли, Изв. РАН. Сер. матем., 1998, том 62, выпуск 5, 103-116

DOI: https://doi.org/10.4213/im217

Использование Общероссийского математического портала Math-Net.Ru подразумевает, что вы прочитали и согласны с пользовательским соглашением http://www . mathnet.ru/rus/agreement

Параметры загрузки:

IP: 52.90 .164 .192

26 апреля 2023 г., 05:05:56 
УДК 512.8

\author{
М.В. Зайцев, С. П. Мищенко
}

\title{
Критерий полиномиальности роста многообразий супералгебр Ли
}

\begin{abstract}
В статье получено описание многообразий супералгебр Ли над полем нулевой характеристики, функция роста которых асимптотически ограничена полиномом некоторой степени.

Библиограффия: 14 наименований.
\end{abstract}

\section{§1. Введение}

В статье изучается рост многообразий супералгебр Ли над полем нулевой характеристики. Напомним необходимые определения. Пусть $F$ - свободная неассоциативная алгебра, порожденная счетным множеством $X=\left\{x_{1}, x_{2}, \ldots\right\}$ над полем $\Phi, \operatorname{char} \Phi=0$, a $\mathbf{V}$ - некоторое многообразие $\Phi$-алгебр. Тогда совокупность тождеств $\mathbf{V}$ образует идеал $I$ в $F$. Обозначим через $P_{n}$ подпространство полилинейных элементов от $x_{1}, \ldots, x_{n}$ в $F$. Положим

$$
\gamma_{n}(\mathbf{V})=\operatorname{dim} \frac{P_{n}}{P_{n} \cap I}
$$

Последовательность $\left(\gamma_{n}(\mathbf{V})\right), n=1,2, \ldots$, является числовой характеристикой многообразия $\mathbf{V}$. Рост $\mathbf{V}$ называют полиномиальным, если $\gamma_{n}(\mathbf{V})<n^{k}$ при достаточно больших $n$, и әкспоненциальным, если $a^{n}<\gamma_{n}(\mathbf{V})<b^{n}$ для некоторых $a, b>1$ при больших $n$.

Если $\gamma_{n}(\mathbf{V})$ асимптотически больше $a^{n}$ для любого заданного $a$, то $\mathbf{V}$ принято называть многообразием сверхәкспоненииального роста. Например, если $\mathbf{V}-$ многообразие всех ассоциативных $\Phi$-алгебр Ли, то $\gamma_{n}(\mathbf{V})=n$ ! Если же $\mathbf{V}$ - многообразие всех алгебр Ли, то $\gamma_{n}(\mathbf{V})=(n-1)$ ! Хорошо известен результат А. Регева [1], согласно которому $\gamma_{n}(\mathbf{V})<a^{n}$ для некоторого $a>1$, если $\mathbf{V}$ - нетривиальное многообразие ассоциативных алгебр. В классе лиевских многообразий реализуются и сверхэкспоненциальные функции роста (см., например, [2], [3]). Однако общим для ассоциативного и лиевского случаев оказалось отсутствие многообразий промежуточного роста, т.е. таких, что функция $\gamma_{n}(\mathbf{V})$ растет быстрее любого полинома, но медленней любой экспоненты (см., например, [4], [5]). К настояшему времени многообразия полиномиального роста описаны и классифицированы как в ассоциативном случае [6], так и в лиевском [7], [8].

В данной статье изучается рост многообразий супералгебр Ли. Основным результатом является описание многообразий полиномиального роста на языке тождеств.

Работа частично поддержана грантом РФФИ, № 96-01-00146. 
ТЕОРема 1. Многообразие $\mathbf{V}$ супералгебр Ли над полем нулевой характеристики имеет полиномиальный рост тогда и только тогда, когда выполняются следующие условия:

1) $\mathbf{V} \subset \mathbf{N}_{c} \mathbf{A}$;

2) существует такое $k$, что любой полилинейный полином, содержащий не менее $k$ четных и $k$ нечетных переменных, тождественно равен нулю ${ }_{8} \mathbf{V}$;

3) в $\mathbf{V}$ для любого $r=0,1, \ldots, c-1$ выполняются тождества вида

$$
\begin{aligned}
& \left(z_{1} z_{2} x_{1} \ldots x_{N}\right) t_{1} \ldots t_{r}\left(z_{3} z_{4} x_{N+1} \ldots x_{2 N}\right) \equiv \\
& \quad \equiv \sum_{\sigma} a_{\sigma}^{r}\left(z_{1} z_{2} x_{\sigma(1)} \ldots x_{\sigma(N)}\right) t_{1} \ldots t_{r}\left(z_{3} z_{4} x_{\sigma(N+1)} \ldots x_{\sigma(2 N)}\right)
\end{aligned}
$$

в котором сумма берется лишь по тем подстановкам $\sigma \in S_{2 N}$, для которьх $\sigma(\{1, \ldots, N\}) \nsubseteq\{1, \ldots, N\}$.

Показано также, что не существует многообразий супералгебр Ли, рост которых больше любого полинома, но меньше любой экспоненты.

ТЕОРема 2. В классе супералгебр Ли отсутствуют многообразия промежуточного роста.

Договоримся об обозначениях. Через $\mathbf{N}_{c}$ мы будем обозначать многообразие, состоящее из супералгебр Ли $L$, для которых $L^{c+1}=0$. При $c=1$ мы получаем многообразие $\mathbf{N}_{1}=\mathbf{A}$ всех абелевых супералгебр Ли. Для двух многообразий $\mathbf{U}$ и V через UV мы будем обозначать их произведение.

\section{§2. Нильпотентность коммутанта супералгебр Ли с полиномиальным ростом тождеств}

В супералгебрах Ли мы будем рассматривать как градуированные (или однородные) тождества, каждая переменная в которых должна быть либо из $\mathscr{L}_{0}$, либо из $\mathscr{L}_{1}$, так и неградуированные. Ясно, что неградуированное полилинейное тождество равносильно некоторой системе градуированных.

Мы будем опускать скобки в неассоциативных произведениях в случае их левонормированной расстановки. Все необходимые сведения о супералгебрах Ли можно найти, например, в [10].

Если $\mathbf{V}$ - многообразие $\Phi$-супералгебр Ли, то можно рассмотреть еще одну числовую характеристику. Обозначим через $\mathscr{L}=\mathscr{L}_{0} \oplus \mathscr{L}_{1}$ свободную супералгебру Ли над $\Phi$ с тем же множеством $X$ четных и множеством $Y=\left\{y_{1}, y_{2}, \ldots\right\}$ нечетных порождающих. Обозначим через $R$ неоднородную подалгебру в $\mathscr{L}$, порожденную элементами $z_{i}=x_{i}+y_{i}, i=1,2, \ldots$, а через $\bar{P}_{n}$ - подпространство полилинейных полиномов от $z_{1}, \ldots, z_{n}$ в $R$. Любое отображение $x_{i} \mapsto a_{i} \in L_{0}, y_{i} \mapsto b_{i} \in L_{1}$ со значениями в некоторой супералгебре Ли $L=L_{0} \oplus L_{1}$ однозначно продолжается до гомоморфизма $R \rightarrow L$. Если $L$ - свободная алгебра счетного ранга многообразия $\mathbf{V}$, то пересечение $J$ ядер всех гомоморфизмов из $R$ в $L$ можно также интерпретировать как идеал тождеств многообразия $\mathbf{V}$ и рассматривать вместо $\gamma_{n}(\mathbf{V})$ коразмерности $\bar{P}_{n} \cap J$ в $\bar{P}_{n}$. Однако эти величины совпадают, поскольку для канонического эпиморфизма $\pi: F \rightarrow R$, где $\pi\left(x_{i}\right)=z_{i}$, выполняются равенства

$$
\pi\left(P_{n}\right)=\bar{P}_{n}, \quad \pi(I)=J, \quad \pi^{-1}(J)=I .
$$


Это замечание позволяет проводить вычисления как в свободной неассоциативной алгебре, так и в свободной супералгебре Ли.

Получим сначала ряд необходимых условий полиномиальности роста.

Лемма 1. Пусть для многообразия $\mathbf{V}$ супералгебр Ли над полем $\Phi$ существует такое натуральное $n$, что $\gamma_{n+1}(\mathbf{V})<2^{\left[\frac{n}{2}\right]}$. Кроме того, пусть $(\operatorname{ad} z)^{m} \equiv 0-$ тождество многообразия $\mathbf{V}$ для нечетной переменной $y$. Тогда для некоторого с выполняется включение $\mathbf{V} \subset \mathbf{N}_{c} \mathbf{A}$.

Квадратные скобки означают целую часть числа.

ДокАЗАТЕльСТво. Пусть $H_{r}^{(k)}=\left\langle\delta_{1}, \ldots, \delta_{r}\right\rangle$ - абелева подгруппа симметрической групшы $S_{2 k}$, порожденная транспозициями $\delta_{i}=(2 i-1,2 i), i=1, \ldots, r$. Будем считать, что группа $H_{k}^{(k)}$, где $k=\left[\frac{n}{2}\right]$, естественньм образом вложена в $S_{n}$.

Рассмотрим элементы супералгебры $L$ вида

$$
z_{0} z_{\sigma(1)} z_{\sigma(2)} \cdots z_{\sigma(n)}, \quad \sigma \in H_{k}^{(k)} .
$$

Так как порядок группы $H_{k}^{(k)}$ равен $2^{k}$, а $\gamma_{n+1}(\mathbf{V})<2^{k}$, то существуют некоторые коэффициенты $a_{\sigma}$, не все равные нулю и такие, что соотношение

$$
\sum_{\sigma \in H_{k}^{(k)}} a_{\sigma} z_{0} z_{\sigma(1)} z_{\sigma(2)} \cdots z_{\sigma(n)}=0
$$

является неградуированным тождеством многообразия $\mathbf{V}$. Без ограничения общности можно считать, что число $n$ является четным. Если это не так, мы просто перейдем к рассмотрению следствия на единицу большей степени.

Если для некоторого $\sigma a_{\sigma} \neq-a_{\sigma \delta_{k}}$, то из (1) получаем нетривиальное следствие:

$$
\sum_{\sigma \in H_{k-1}^{(k)}} a_{\sigma}^{\prime} z_{0} z_{\sigma(1)} \cdots z_{\sigma(2 k-2)} t t=0,
$$

линеаризация которого по переменной $t$ имеет вид

$$
\sum_{\sigma \in H_{k-1}^{(k)}} a_{\sigma}^{\prime} z_{0} z_{\sigma(1)} \cdots z_{\sigma(2 k-2)}\left(z_{2 k} z_{2 k-1}+z_{2 k-1} z_{2 k}\right)=0 .
$$

Если же для всех $\sigma \in H_{k}^{(k)} a_{\sigma}=-a_{\sigma \delta_{k}}$, то тождество (1) можно переписать так:

$$
\sum_{\sigma \in H_{k-1}^{(k)}} a_{\sigma} z_{0} z_{\sigma(1)} \cdots z_{\sigma(2 k-2)}\left(z_{2 k} z_{2 k-1}-z_{2 k-1} z_{2 k}\right)=0 .
$$

Продолжив этот процесс для $\delta_{i}, i=k-1, \ldots, 1$, получим, что в многообразии V выполнено тождество

$$
z_{0}\left(Z_{2} Z_{1}+c_{1} Z_{1} Z_{2}\right)\left(Z_{4} Z_{3}+c_{2} Z_{3} Z_{4}\right) \cdots\left(Z_{2 k} Z_{2 k-1}+c_{k} Z_{2 k-1} Z_{2 k}\right)=0,
$$

в котором числа $c_{1}, \ldots, c_{k}$ равны 1 или -1, a $Z_{i}=\operatorname{ad} z_{i}$. 
Доказательство проведем индукцией по числу $k$. Пусть вместо переменной $z_{0}$ стоит левонормированное произведение коммутаторов, которое обозначим через $T$. С каждым шагом индукции число скобок будет уменьшаться, а число сомножителей произведения $T$ расти.

Итак, пусть в многообразии $\mathbf{V}$ выполнено следующее тождество:

$$
T z_{1} z_{2} D=a T z_{2} z_{1} D
$$

в котором число $a$ равно 1 или $-1, T$ является левонормированным произведением коммутаторов, а $D=\left(Z_{4} Z_{3}+c_{2} Z_{3} Z_{4}\right) \ldots\left(Z_{2 k} Z_{2 k-1}+c_{k} Z_{2 k-1} Z_{2 k}\right)$.

При получении следствий из тождества (2) можно подставлять любые элементы вместо переменных $z_{1}$ и $z_{2}$, а также подставить вместо $T$ произведение $T z$. Поясним последнее утверждение. Тождество

$$
T z z_{1} z_{2} D=a T z z_{2} z_{1} D
$$

является следствием тождества (2), так как переменной $z$ можно продифференцировать произведение $T$, получив сумму произведений такого же количества коммутаторов. Таким образом, все слагаемые будут следствиями тождества (2).

Для осуществления индуктивного перехода достаточно получить следствие вида $T_{1} D=0$, где $T_{1}$ - левонормированное произведение коммутаторов.

В силу тождества (3) из шести элементов, получаемых перестановкой переменных $z_{1}, z_{2}, z_{3}$, остаются три. Переобозначим их:

$$
s_{1}=T z_{1} z_{3} z_{2} D, \quad s_{2}=T z_{2} z_{3} z_{1} D, \quad s_{3}=T z_{3} z_{2} z_{1} D .
$$

Будем дополнительно считать, что $\alpha, \beta, \gamma$ - четности переменных $z_{1}, z_{2}, z_{3}$ соответственно. Тогда из тождества (2) подстановкой коммутатора вместо одной переменной и переобозначением переменных получим систему линейных уравнений относительно введенных переменных:

$$
\begin{aligned}
\left((-1)^{\beta \gamma}-a\right) s_{1}-(-1)^{\beta \gamma} s_{2}+s_{3} & =0 \\
-(-1)^{\alpha \gamma} s_{1}+\left((-1)^{\alpha \gamma}-a\right) s_{2}+a s_{3} & =0 \\
s_{1}-(-1)^{\alpha \beta} s_{2}+\left((-1)^{\alpha \beta}-a\right) s_{3} & =0 .
\end{aligned}
$$

Рассмотрим случай, когда в тождестве (2) коэффициент $a$ равен 1. Перебирая все возможные варианты четностей переменных $z_{1}, z_{2}, z_{3}$ в системе (4), получаем равенство $s_{2}=s_{3}$. Следовательно, в многообразии $\mathbf{V}$ вьполнено неградуированное тождество $T z_{2} z_{1} z D=T z_{1} z_{2} z D$. Увеличивая "хвост" и повторяя процедуру, получаем, что в многообразии для любой перестановки $p$ выполнено тождество

$$
T z_{p(1)} \cdots z_{p(s)} D=T z_{1} \cdots z_{s} D .
$$

Рассмотрим элемент

$$
T\left(z_{11} z_{12}\right) \cdots\left(z_{k 1} z_{k 2}\right) D
$$

Докажем, что при достаточно большом значении числа $k$ он равен нулю тождественно.

Если есть хотя бы одна четная переменная, то сначала раскроем все скобки, кроме коммутатора с этой переменной. Раскрывая этот коммутатор, получим нуль 
в силу тождества (5). Пусть теперь все переменные нечетные. Соотношение (5) выполняется при всех $s$, в том числе при $s=m$, для которого $(\operatorname{ad} z)^{m}=0$ для нечетных $z$. Линеаризуя тождество $T(\operatorname{ad} z)^{m} D=0$ и учитывая (5), получаем $T z_{1} \cdots z_{m} D=0$. Раскрывая все коммутаторные скобки в (6) от переменных $z_{i j}$, мы получаем нуль при $k>\frac{m}{2}$.

Осталось рассмотреть случай, когда в тождестве (2) выполняется равенство $a=-1$. Перебирая все возможные варианты четностей переменных системы (4), получаем, что в нашем многообразии выполнена серия градуированных тождеств $T z_{3} z_{2} z_{1} D=b T z_{2} z_{3} z_{1} D$, где коэффициент $b$ зависит от четностей переменных $z_{1}$, $z_{2}, z_{3}$. Более того, если две или три переменные из $z_{1}, z_{2}, z_{3}$ являются четными, то коэффициент $b$ равен нулю, т.е. выполнены такие градуированные тождества:

$$
T z_{3} z_{2} z_{1} D=0 \text {. }
$$

Докажем, что следствием выписанных градуированных тождеств и тождества (2) является неградуированное тождество

$$
T\left(z_{1} z_{2}\right)\left(z_{3} z_{4}\right) D=0 .
$$

Если в одной из скобок обе переменные нечетные, то, переставляя этот коммутатор вправо и раскрывая его, получим нуль в силу тождества (3). Пусть один из коммутаторов составлен из двух четных переменных; тогда получим нуль в силу серии градуированных тождеств (7). Осталось рассмотреть случай, когда каждый из коммутаторов составлен из одной четной и одной нечетной переменной. Обозначим четные переменные через $x$, а нечетные - через $y$. Тогда верна такая цепочка равенств:

$$
T\left(y_{4} x_{3}\right)\left(x_{1} y_{2}\right) D=(1-b) T y_{4} x_{3}\left(x_{1} y_{2}\right) D=0 .
$$

Последнее равенство получено как следствие серии тождеств (7), так как переменной $y_{4}$ можно продифференщировать произведение коммутаторов $T$, получив сумму произведений коммутаторов. Мы уже применяли этот прием при выводе тождества (3) из тождества (2). Итак, в рассматриваемом случае, когда в тождестве (2) коэффициент $a$ равен -1 , в многообразии $\mathbf{V}$ элемент (6) уже при $k=2$ тождественно равен нулю.

Таким образом, в обоих случаях элемент (6) является тождественно нулевым, т.е. в многообразии $\mathbf{V}$ выполнено тождество необходимого вида $T_{1} D=0$, где $T_{1}=T\left(z_{11} z_{12}\right) \ldots\left(z_{k 1} z_{k 2}\right)$.

Доказательство возможности индуктивного шага завершено. Лемма 1 полностью доказана.

Обозначим через $H=H_{0} \oplus H_{1}$ четырехмерную супералгебру Ли с базисом $a$, $b, u, v$ и таблицей умножения $a b=0, u v=0, a u=v, a v=u, b u=u, b v=v$, $a^{2}=2 b, b^{2}=u^{2}=v^{2}=0$, в которой $H_{0}=\langle b, v\rangle, H_{1}=\langle a, u\rangle$. Очевидно, что $(\operatorname{ad} b)^{n}$ - ненулевое отображение на $H$ при любом $n$, поэтому в $H$ не выполняется тождество $(\operatorname{ad} z)^{n} \equiv 0$ для нечетной переменной $z$ ни при каком $n$.

Лемма 2. Для многообразия супералгебр Ли $\mathbf{V}$ следующие условия әквивалентния:

1) ни для какого $n$ в $\mathbf{V}$ не выполняется тождество вида $(\operatorname{ad} y)^{n} \equiv 0$, $y \in \mathscr{L}_{1}$

2) $\mathbf{V}$ содержит супералгебру $H$. 
ДокаЗАТЕЛЬСТво. Очевидно, что второе условие влечет первое. Пусть теперь тождество $(\operatorname{ad} y)^{n} \equiv 0$ не выполняется в $\mathbf{V}$ для нечетной переменной $y$. Обозначим через $M$ свободную $\mathbf{V}$-алгебру с одним четным порождающим $x$ и двумя нечетными $y_{1}$ и $y_{2}$. Тогда $\left(\operatorname{ad} y_{1}\right)^{n} \neq 0$. Поскольку в свободной супералгебре Ли $\mathscr{L}$ нет элементов степени 1 по $x_{1}$ и степени $n$ по $y_{1}$, лежаших в $\left(\mathscr{L}^{3}\right)^{2}$, то в факторалгебре $L=M /\left(M^{3}\right)^{2}=L_{0} \oplus L_{1}$ также не вьполняется тождество $(\operatorname{ad} y)^{n} \equiv 0$, и $L \in \mathbf{V} \cap \mathbf{A} \mathbf{N}_{2}$. Так как у $L$ лишь один нечетный порождаюший, то $L_{0}^{2} \subset L^{3}$, следовательно,

$$
\left[\left[L, L_{0}^{2}\right], L_{0}^{2}\right]=0 .
$$

Из соотношения (8) и включения $L \in \mathbf{A N}_{2}$ следует, что супералгебра $L$ представима и поэтому вложима в декартово произведение некоторого семейства супералгебр $L_{\alpha}$, размерности которых ограничены некоторой общей константой $N$ (см. [11,гл.6] или [12]). При этом все $L_{\alpha}$ являются гомоморфными образами $L$ и поэтому лежат в $\mathbf{V}$. Отсюда следует, что в $L$ найдется идеал $J$ конечной коразмерности, лежащий в $L^{3}$, для которого ad $y_{1}$ действует на $L^{3} / J$ ненильпотентно.

Обозначим через $T=T_{0} \oplus T_{1}$ конечномерный $L$-модуль $L^{3} / J$ и через $\phi$ - ненильпотентный оператор $\left(\operatorname{ad} y_{1}\right)^{2}$ на $T$. Предположим сначала, что поле $\Phi$ алгебраически замкнуто. Тогда существует ненулевой элемент $c$ из $T_{0}$, для которого $\phi(c)=\lambda c$ и $\lambda \neq 0$. Положим $y=\lambda^{-\frac{1}{2}} y_{1}$ и рассмотрим подалгебру $B$ в полупрямом произведении $T \lambda L=P$, порожденную $y$ и $c$. Несложньм упражнением является доказательство того, что $P \in \mathbf{V}$. Пусть $d=y c, e=\frac{1}{2} y^{2}$. Тогда $B=\langle e, y, c, d\rangle$, $B_{0}=\langle e, c\rangle, B_{1}=\langle y, d\rangle$. Элементы $y, c, e$ и $d$ перемножаются следуюшим образом: $y e=0, d c=0, y d=c, y c=d, e d=d, e c=c, y^{2}=2 e, e^{2}=c^{2}=d^{2}=0$. Элементы $y, e, c, d$ линейно независимы, поскольку $y, c \in T_{0}, e, d \in T_{1}$. Следовательно, $B$ изоморфна $H$, и для алгебраически замкнутого поля лемма доказана. Если же $\Phi$ незамкнуто, то достаточно присоединить к нему один из характеристических корней оператора $\phi$, расширить алгебру $T \lambda L=P$ и взять в ней аналогичную четырехмерную подалгебру. Лемма 2 доказана.

Для доказательства основных результатов статьи нам потребуются оценки роста многообразия var $H$.

ЛЕмма 3. Для любой константы $\alpha<\frac{2}{3}$ выполняются неравенства $2^{\alpha n} \leqslant$ $\gamma_{n}(\operatorname{var} H) \leqslant n^{2} 2^{n}$.

ДокАЗАТЕЛЬСТво. Получим сначала нижнюю оценку роста. Пусть $z_{0}, z_{1}$, $z_{2}, \ldots, x_{1}, x_{2}, \ldots$ - переменные из свободной неассоциативной алгебры $F$. Построим семейство полилинейных полиномов $f_{0}, f_{1}, \ldots$ из $F$, положив $f_{0}=z_{0}$, $f_{1}=z_{1}\left(\left(x_{1} f_{0}\right) x_{2}\right), \ldots, f_{k}=f_{k}\left(z_{0}, \ldots, z_{k}, x_{1}, \ldots, x_{2 k}\right)=z_{k}\left(\left(x_{2 k-1} f_{k-1}\right) x_{2 k}\right)$. Обозначим через $D=D(k)$ таблицу Юнга, состоящую из двух строк длины $k$, в первой строке которой стоят числа $1,3, \ldots, 2 k-1$, а во второй числа $2,4, \ldots, 2 k$. Если $e_{D}=\sum(-1)^{q} p q$ - соответствуюший таблице $D$ элемент группового кольца групшы подстановок $S_{2 k}$, то $e_{D} \circ f_{k}=g_{k}=g_{k}\left(z_{0}, z_{1}, \ldots, z_{k}, x_{1}, \ldots, x_{2 k}\right)$ - полилинейный полином степени $3 k+1$ в $F$. Он равен сумме

$$
g_{k}=g_{k}^{1}+\cdots+g_{k}^{N},
$$

в которой $N=2^{k}(k !)^{2}$, а каждый $g_{k}^{i}$ - одночлен вида $(-1)^{q} p q \circ f_{k}$, где $q$ принадлежит подгруппе в $S_{2 k}$, порожденной транспозициями $(1,2), \ldots,(2 k-1,2 k)$, a $p \in S(A) \times S(B), A=\{1,3, \ldots, 2 k-1\}, B=\{2,4, \ldots, 2 k\}$. 
Вычислим значение $g_{k}^{i}=(-1)^{q} p q \circ f_{k}$ в алгебре $H$ на элементах $z_{0}=u$, $z_{1}=\cdots=z_{k}=a, x_{1}=a, x_{2}=b, \ldots, x_{2 k-1}=a, x_{2 k}=b$. Это значение рав$\mathrm{HO}$

$$
(-1)^{q}(-1)^{q} f_{k}(u, \underbrace{a, \ldots, a}_{k}, a, b, a, b, \ldots, a, b)=(-1)^{k} u .
$$

Следовательно,

$$
g_{k}(u, a, \ldots, a, a, b, \ldots, a, b)=(-1)^{k} 2^{k}(k !)^{2} \neq 0,
$$

т.е. $g_{k}=g_{k}\left(z_{0}, \ldots, z_{k}, x_{1}, \ldots, x_{2 k}\right)$ не является тождеством алгебры $H$. Действуя на переменные $x_{1}, \ldots, x_{2 k}$ элементами симметрической групшы $S_{2 k}$, мы получим в полилинейной компоненте $P_{3 k+1}$ алгебры $F$ подпространство размерности не меньше, чем размерность левого идеала $R e_{D}$ в групповом кольце $R=\Phi S_{2 k}$. Хорошо известно (см., например, [13]), что

$$
\operatorname{dim} \operatorname{Re}_{D}=\frac{(2 k) !}{k !(k+1) !}
$$

Используя формулу Стирлинга, легко показать, что величина (9) при больших $k$ превосходит $\frac{A}{\sqrt{k}} 2^{2 k}$, где $A$ - некоторая константа. Поскольку степень полинома $g_{k}$ равна $3 k+1$, то $k=\frac{n-1}{3}$. Отсюда получается оценка $\gamma_{n}(\operatorname{var} H)>2^{\alpha n}$ для любого $\alpha<\frac{2}{3}$, и первая часть леммы доказана.

Чтобы получить верхнюю оценку роста, рассмотрим свободную неассоциативную алгебру $\widetilde{F}=\widetilde{F}_{0} \oplus \widetilde{F}_{1}$ с $\mathbf{Z}_{2}$-градуировкой, которая порождена элементами $x_{1}^{0}, x_{2}^{0}, \cdots \in \widetilde{F}_{0}, x_{1}^{1}, x_{2}^{1}, \cdots \in \widetilde{F}_{1}$. Можно считать, что $F$ вложена в $\widetilde{F}$, если положить $x_{i}=x_{i}^{0}+x_{i}^{1}$. Пусть теперь $P_{n}-$ пространство полилинейных многочленов от $x_{1}, \ldots, x_{n}$ в $F$, а $P_{n}(t)$ - пространство полилинейных многочленов от $x_{1}^{t_{1}}, \ldots, x_{n}^{t_{n}}$ в $\widetilde{F}$, где $t=\left(t_{1}, \ldots, t_{n}\right) \in \mathbf{Z}_{2}^{n}$. Тогда

$$
P_{n} \subset \sum_{t \in \mathbf{Z}_{2}^{n}} P_{n}(t),
$$

и если $I$ - идеал тождеств алгебры $H$ в $F$, то

$$
\frac{P_{n}+I}{I} \subset \sum_{t \in \mathbf{Z}_{2}^{n}} \frac{P_{n}(t)+I}{I}
$$

следовательно,

$$
\gamma_{n}(\operatorname{var} H)=\operatorname{dim} \frac{P_{n}}{P_{n} \cap I} \leqslant \sum_{t \in \mathbf{Z}_{2}^{n}} \operatorname{dim} \frac{P_{n}(t)}{P_{n}(t) \cap I} .
$$

Количество слагаемых в правой части (10) равно $2^{n}$. Поэтому достаточно показать, что

$$
\operatorname{dim} \frac{P_{n}(t)}{P_{n}(t) \cap I} \leqslant n^{2}
$$

при любом $t \in \mathbf{Z}_{2}^{n}$. 
Элементы из $P_{n}(t) \cap I$ являются градуированными тождествами алгебры $L$. Рассмотрим $t=\left(t_{1}, \ldots, t_{n}\right) \in \mathbf{Z}_{2}^{n}$ и все полилинейные полиномы от $x_{1}^{t_{1}}, \ldots, x_{n}^{t_{n}}$ в $\widetilde{F}$. Отметим, что для любых однородных элементов $c_{1}, \ldots, c_{5}$ из $H$ выполняется равенство

$$
c_{1} c_{2} c_{3} c_{4} c_{5}=c_{1} c_{2} c_{3} c_{5} c_{4} \text {. }
$$

Из (11) и определяюших тождеств супералгебры Ли следует, что любой полилинейный полином от $x_{1}^{t_{1}}, \ldots, x_{n}^{t_{n}}$ по модулю $I$ равен линейной комбинации произведений

$$
x_{1}^{t_{1}} x_{i}^{t_{i}} x_{j}^{t_{j}} X_{i j}
$$

где через $X_{i j}$ сокращенно обозначено последовательное умножение справа на все оставшиеся $x_{k}^{t_{k}}$ в порядке возрастания индекса $k$. Количество элементов (12) равно $n^{2}$, поэтому размерность $P_{n}(t)$ по модулю $I$ не выше $n^{2}$, и лемма 3 доказана.

Из лемм 1,2 и 3 вытекает включение $\mathbf{V} \subset \mathbf{N}_{c} \mathbf{A}$ для любого многообразия супералгебр Ли полиномиального роста.

\section{§3. Необходимые условия полиномиального роста многообразия}

Лемма 4. Пусть $\mathbf{V}$ - многообразие супералгебр Ли, лежащее в $\mathbf{N}_{c} \mathbf{A}$, рост которого не превышает $2^{\frac{n}{4 c}}$. Тогда существует такое $N$, что любой полилинейный полином $f\left(x_{1}, \ldots, x_{k}, y_{1}, \ldots, y_{m}\right)$ от $x_{1}, \ldots, x_{k} \in \mathscr{L}_{0}, y_{1}, \ldots, y_{m} \in \mathscr{L}_{1}$ равен нулю тожсдественно в $\mathbf{V}$, если $k, m \geqslant N$.

ДокАЗАТЕЛЬСтво. Сначала мы докажем, что существует такое $N_{0}$, что $f\left(x_{1}\right.$, $\left.\ldots, x_{k}, y_{1}, \ldots, y_{m}\right) \equiv 0$ в $\mathbf{V}$, если $n=m+k \geqslant N_{0}$ и $k, m>\frac{2}{5} n$. Воспользуемся индукцией по $c$. Пусть $c=1$. Рассмотрим относительно свободную алгебру $L=$ $L_{0} \oplus L_{1}$ многообразия $\mathbf{V}$ с четными образующими $\tilde{x}_{1}, \tilde{x}_{2}, \ldots$ и нечетными $\tilde{y}_{1}, \tilde{y}_{2}, \ldots$ Предположим, что наше предположение неверно. Тогда при любом $n$ найдутся такие нечетные $m$ и $k$, что $m, k>\frac{1}{3} n$ и полилинейньй одночлен

$$
A=z \tilde{x}_{1} \ldots \tilde{x}_{k} \tilde{y}_{1} \ldots \tilde{y}_{m}
$$

не равен нулю в $L$, где $z=z_{1} \ldots z_{t}$-произведение некоторых порождающих $\tilde{x}_{i}, \tilde{y}_{j}$, причем $t>1$. Возьмем в свободной неассоциативной алгебре $F$ левонормированный одночлен $f=x_{k+m+1} \ldots x_{n} x_{1} \ldots x_{k} x_{k+1} \ldots x_{m+k}$. Пусть, например, $k \leqslant m$. Рассмотрим таблищу Юнга $D$ из $2 k-1$ клеток, состоящую из одной строки длины $k$ и одного столбца высоты $k$. В строке стоят числа $1, \ldots, k$, а в столбце числа $1, k+1, \ldots, 2 k-1$. Рассмотрим элемент группового кольца симметрической группы $S_{2 k-1}$

$$
e_{D}=\sum_{p \in G_{r}, q \in G_{c}}(-1)^{q} p q,
$$

где $G_{c}$ - подгруппа в $S_{2 k-1}$, оставляющая на месте символы $2, \ldots, k$, a $G_{r}$ - подгруппа, оставляющая неподвижными $k+1, \ldots, 2 k-1$. Положим теперь $g=$ $g\left(x_{1}, \ldots, x_{n}\right)=e_{D} \circ f$ и покажем, что полином $g$ не равен нулю тождественно в $L$. Запишем $g$ в виде суммы одночленов $g=g_{1}+\cdots+g_{s}$ вида $(-1)^{q} p q \circ f$ и рассмотрим гомоморфизм $\phi$ из $F$ в $L$, переводящий $x_{1}, \ldots, x_{k}$ в $\tilde{x}_{1}, \ldots, \tilde{x}_{k}, x_{k+1}, \ldots, x_{k+m}$ в $\tilde{y}_{1}, \ldots, \tilde{y}_{m}$, а $x_{k+m+1}, \ldots, x_{n}$ в $z_{1}, \ldots, z_{t}$. Пусть $p, q$ - произвольные подстановки из $G_{c}, G_{r}$ соответственно и $g^{\prime}=(-1)^{q} p q \circ f$. Вычислим значение $\phi\left(g^{\prime}\right)$ в $L$. 
Заметим, что элемент $A$ из (13) симметричен по переменным $\tilde{x}_{1}, \ldots, \tilde{x}_{k}$ и кососимметричен по переменным $\tilde{y}_{1}, \ldots, \tilde{y}_{m}$ (поскольку $c=1$ ). Поэтому если $q(1)=1$, то $\phi\left(g^{\prime}\right)=A$. Пусть $q(1) \neq 1$. Тогда $q(k+j)=1$ при некотором $1 \leqslant j \leqslant k-1$. Тогда

$$
\begin{aligned}
g^{\prime}= & (-1)^{q} x_{k+m+1} \ldots x_{n} x_{q(1)} x_{p(2)} \ldots x_{p(k)} x_{q(k+1)} \ldots \\
& \ldots x_{q(k+j-1)} x_{p(1)} x_{q(k+j+1)} \ldots x_{q(2 k-1)}
\end{aligned}
$$

и $\phi\left(g^{\prime}\right)=(-1)^{j-1} A$. Количество подстановок $q \in G_{c}$, для которых $q^{-1}(1)=k+j$, равно $(k-1)$ ! и не зависит от $j=1, \ldots, k-1$. Поскольку число $k$ можно считать нечетным, все слагаемые вида $\phi\left(g^{\prime}\right)$, у которых $q(1) \neq 1$, в сумме $\phi(g)=\phi\left(g_{1}\right)+$ $\cdots+\phi\left(g_{s}\right)$ сокрашаются, поэтому $\phi(g)=k !(k-1) ! A \neq 0$.

Мы доказали, что в $L$ не выполняется тождество $g \equiv 0$, где $g=e_{D} \circ f$. Следовательно, коразмерность идеала тождеств многообразия $\mathbf{V}$ в $P_{n}$ не меньше размерности левого идеала $R e_{D}$ группового кольца $R=\Phi S_{2 k-1}$, которая равна

$$
\frac{(2 k-1) !}{k ! k !}=\tau(k)
$$

(см. [13]). Используя формулу Стирлинга, легко получить оценку

$$
\tau(k)>\frac{1}{\sqrt{k}} 2^{2 k}>\frac{1}{\sqrt{n}} 2^{\frac{2}{3} n}>2^{\frac{n}{4}}
$$

для достаточно больших $n \geqslant N_{0}$. Отсюда и следует, что при $c=1$ любой одночлен степени $n \geqslant N_{0}$, содержаший не менее $\frac{2}{5} n$ четных и нечетных сомножителей одновременно, равен нулю в $L$.

Пусть теперь $c>1$. По предположению индукции можно считать, что найдется $N_{0}$, при котором для всех $n \geqslant N_{0}$ одночлен, содержащий не менее $\frac{2}{5} n$ четных и нечетных сомножителей, лежит в $\left(L^{2}\right)^{c}$. Для таких $n$ рассмотрим одночлены из $\left(L^{2}\right)^{c}$, содержашие более $\frac{2}{5} n$ как четных, так и нечетных переменных. Если среди них есть ненулевой, то он представляет собой произведение $c$ левонормированных скобок-одночленов, поэтому в одной из скобок будет более $\frac{2 n}{5 c}$ нечетных переменных и в одной - более $\frac{2 n}{5 c}$ четных. Если это одна и та же скобка, то она имеет вид $z_{1} \ldots z_{t} \tilde{x}_{1} \ldots \tilde{x}_{k} \tilde{y}_{1} \ldots \tilde{y}_{k-1}$, где $\tilde{x}_{1} \ldots \tilde{x}_{k} \in L_{0}, \tilde{y}_{1} \ldots \tilde{y}_{k-1} \in L_{1}, k$ четно и $k>\frac{n}{3 c} . \mathrm{B}$ этом случае, как и при $c=1$, доказывается, что рост многообразия $\mathbf{V}$ не меньше, чем $\frac{1}{\sqrt{k}} 2^{2 k}>\frac{1}{\sqrt{n}} 2^{\frac{2 n}{3 c}}>2^{\frac{n}{4 c}}$, что противоречит условию леммы.

Рассмотрим теперь ситуацию, ког да эти скобки различны. Для наглядности мы будем считать, что это две крайние справа скобки, т.е. ненулевой элемент из $\left(L^{2}\right)^{c}$ имеет вид

$$
A_{1} \ldots A_{c-2}\left(z_{1} \tilde{x}_{1} \ldots \tilde{x}_{k}\right)\left(z_{2} \tilde{y}_{1} \ldots \tilde{y}_{k-1}\right),
$$

где $A_{i}, z_{1}, z_{2} \in L^{2}$ и $k \geqslant \frac{2 n}{5 c}$. Возьмем в свободной неассоциативной алгебре одночлен

$$
f=B_{1} \ldots B_{c-2}\left(d_{1} x_{1} \ldots x_{k}\right)\left(d_{2} x_{k+1} \ldots x_{2 k-1}\right),
$$

у которого $B_{1}, \ldots, B_{c-2}, d_{1}, d_{2}$ - левонормированные одночлены тех же степеней, что и $A_{1}, \ldots, A_{c-2}, z_{1}, z_{2}$ соответственно. Снова возьмем элемент (14) и положим $g=e_{D} \circ f$. Как и прежде, в $L$ должно выполняться тождество $g \equiv 0$, поскольку в противном случае рост многообразия $\mathbf{V}$ будет больше $2^{2 k} \geqslant 2^{\frac{4 n}{5 c}}$. В частности, $g$ должен принимать нулевое значение при гомоморфизме $\phi: F \rightarrow L$, для которого 
$\phi\left(B_{i}\right)=A_{i}, i=1, \ldots, c-2, \phi\left(d_{1}\right)=z_{1}, \phi\left(d_{2}\right)=z_{2}, \phi\left(x_{i}\right)=\tilde{x}_{i}, \phi\left(x_{k+j}\right)=\tilde{y}_{j}, j=$ $1, \ldots, k-1$. Однако равенство $\phi(g)=0$ означает, что в $L$ выполнено соотношение

$$
\begin{aligned}
& A\left(z_{1} \tilde{x}_{1} \ldots \tilde{x}_{k}\right)\left(z_{2} \tilde{y}_{1} \ldots \tilde{y}_{k-1}\right)= \\
& \quad=\sum \lambda_{i j} A\left(z_{1} \tilde{y}_{i} \tilde{x}_{2} \ldots \tilde{x}_{k}\right)\left(z_{2} \tilde{x}_{j} \tilde{y}_{1} \ldots \tilde{y}_{k-1}\right),
\end{aligned}
$$

где $A=A_{1} \ldots A_{c-2}$. Отметим также, что для получения нижней экспоненциальной оценки на рост нам достаточно было считать, что $k \geqslant \frac{n}{6 c}$ у элемента (14). Другими словами, соотношение (16) позволяет нам "перебрасывать" четные переменные в скобку с нечетными, если $k \geqslant \frac{n}{6 c}$. В конце конщов мы выражаем элемент (15) через произведения $c$ левонормированных скобок, в одной из которых не менее $k=\frac{n}{6 c}$ и четных и нечетных переменных одновременно. Но наличие такого ненулевого элемента в $L$ гарантирует рост вьше $\frac{1}{\sqrt{k}} 2^{2 k}>2^{\frac{n}{4 c}}$, что противоречит условию леммы. Таким образом, мы доказали, что при достаточно больших $n \geqslant N_{0}$ любое произведение, в котором одновременно встречаются не менее $\frac{2}{5} n$ как четных, так и нечетных элементов, равно нулю в $L$.

Теперь мы индукцией по $c$ докажем сушествование такого $N$, что $f\left(x_{1}, \ldots, x_{k}\right.$, $\left.y_{1}, \ldots, y_{m}\right) \equiv 0$ в $L$ при четных $x_{1}, \ldots, x_{k}$ и нечетных $y_{1}, \ldots, y_{m}$, если $m, k \geqslant N$. Если $c=1$, то из доказанного выше следует, что при некотором $N$ в $\mathbf{V}$ выполняется система тождеств

$$
z x_{1} \ldots x_{N} y_{1} \ldots y_{N} \equiv 0
$$

в которой $z$ равен $x_{N+1} x_{N+2}, y_{N+1} y_{N+2}$ либо $x_{N+1} y_{N+1}$. Очевидно, что любой полилинейный многочлен $f\left(x_{1}, \ldots, x_{k}, y_{1}, \ldots, y_{m}\right)$ по модулю $\left(\mathscr{L}^{2}\right)^{2}$ является гомоморфным образом одного из элементов из левой части (17), если $k, m \geqslant N+2$, откуда следует утверждение леммы для $c=1$.

Пусть теперь $c>1$. Из индуктивных соображений можно считать, что существует такое $N_{0}$, для которого в относительно свободной алгебре $L$ многообразия $\mathbf{V}$ любой полилинейный полином от $x_{1}, \ldots, x_{k}, y_{1}, \ldots, y_{m}$ лежит в $\left(L^{2}\right)^{c}$ при $k, m>N_{0}$. С другой стороны, как доказано выше, в $\mathbf{V}$ выполняется система полилинейных тождеств вида

$$
\begin{gathered}
z_{1} \ldots z_{i-1}\left(z_{i} x_{1} \ldots x_{N} y_{1} \ldots y_{N}\right) z_{i+1} \ldots z_{c} \equiv 0 \\
z_{1} \ldots z_{i-1}\left(z_{i} x_{1} \ldots x_{N}\right) z_{i+1} \ldots z_{j-1}\left(z_{j} y_{1} \ldots y_{N}\right) z_{j+1} \ldots z_{c} \equiv 0
\end{gathered}
$$

в которых каждый $z_{r}$ равен одному из произведений $x_{\alpha} x_{\beta}, x_{\alpha} y_{\beta}$ или $y_{\alpha} y_{\beta}$, а $i$ и $j$ изменяются от 1 до $c$. Очевидно, что любое полилинейное тождество $f=$ $f\left(x_{1}, \ldots, x_{k}, y_{1}, \ldots, y_{m}\right) \equiv 0$ является следствием тождеств (18), (19), если $f \in$ $\left(\mathscr{L}^{2}\right)^{c}$ и $k, m>(N+1) c$. Таким образом, любой элемент алгебры $L$, в котором число четных и нечетных переменных больше, чем $N_{0}$ и $(N+1) c$, равен нулю, и лемма 4 доказана.

Лемма 5. Пусть $\mathbf{V}$ - многообразие супералгебр Ли, рост которого меньше, чем $2^{\frac{n}{2}}$. Тогда найдется такое $N$, что для любого $r=0,1, \ldots, c-1$ в $\mathbf{V}$ выполняется тождество вида

$$
\begin{aligned}
& \left(z_{1} z_{2} x_{1} \ldots x_{N}\right) t_{1} \ldots t_{r}\left(z_{3} z_{4} x_{N+1} \ldots x_{2 N}\right) \equiv \\
& \quad \equiv \sum_{\sigma, r} \alpha_{\sigma}^{r}\left(z_{1} z_{2} x_{\sigma(1)} \ldots x_{\sigma(N)}\right) t_{1} \ldots t_{r}\left(z_{3} z_{4} x_{\sigma(N+1)} \ldots x_{\sigma(2 N)}\right)
\end{aligned}
$$


в котором сумма берется лишь по тем подстановкам $\sigma \in S_{2 N}$, для которых $\sigma(\{1, \ldots, N\}) \nsubseteq\{1, \ldots, N\}$.

ДоКАЗАТЕЛЬСТВО. Пусть $J$ - подмножество мощности $N$ в $\{1,2, \ldots, 2 N\}$, а $I=\{1,2, \ldots, 2 N\} \backslash J$. Занумеруем элементы в $I$ и $J$ в порядке возрастания: $I=\left\{i_{1}, \ldots, i_{N}\right\}, J=\left\{j_{1}, \ldots, j_{N}\right\}$ и рассмотрим полилинейные одночлены в $F$ вида

$$
d_{r, J}=\left(z_{1} z_{2} x_{j_{1}} \ldots x_{j_{N}}\right) t_{1} \ldots t_{r}\left(z_{3} z_{4} x_{i_{1}} \ldots x_{i_{N}}\right) .
$$

Нетрудно заметить, что они линейно независимы в $F$, а их количество равно

$$
h(N)=C_{2 N}^{N}=\frac{2 N !}{N ! N !} .
$$

Величина $h(N)$ растет быстрее $\frac{1}{\sqrt{N}} 2^{2 N}$, и поскольку $n=\operatorname{deg} d_{r, J}$ и $N$ связаны соотношением $2 N+4 \leqslant n \leqslant 2 N+c+3$, то асимптотически $h(N)>2^{\frac{n}{2}}$. Следовательно, элементы $d_{r, J}$ линейно зависимы по модулю тождеств многообразия $\mathbf{V}$. Равенство нулю их нетривиальной линейной комбинации и означает тождество вида (20) в V. Лемма 5 доказана.

\section{§4. Доказательство основных результатов}

Полученные нами необходимые условия оказываются и достаточными для полиномиальности роста.

ДОКАЗАТЕЛЬСТво ТЕОРЕМЫ 1. Необходимость условий 1),2),3) доказана в леммах 1-5. Пусть теперь $\mathbf{V} \subset \mathbf{N}_{c} \mathbf{A}$ и выполнены условия 2) и 3). Докажем, что $\mathbf{V}$ - многообразие полиномиального роста. Заметим сначала, что, как и в лемме 2 , для многообразия $\mathbf{V}$ верна оценка, аналогичная (10),

$$
\gamma_{n}(\mathbf{V}) \leqslant \sum_{t \in \mathbf{Z}_{2}^{n}} \operatorname{dim} \frac{P_{n}(t)}{P_{n}(t) \cap I},
$$

в которой $I$ - идеал тождеств многообразия $\mathbf{V}$ в алгебре $F$. Условие 2 ) означает, что в правой части (21) число ненулевых слагаемых не превьшает числа

$$
2\left(\left(\begin{array}{c}
n \\
0
\end{array}\right)+\left(\begin{array}{c}
n \\
1
\end{array}\right)+\cdots+\left(\begin{array}{c}
n \\
k-1
\end{array}\right)\right)
$$

которое растет полиномиально с ростом $n$. Поэтому достаточно доказать, что число линейно независимых по модулю $I$ полилинейных элементов в $F$ от фиксированного числа $m<k$, например четных переменных и $(n-m)$ нечетных переменных, растет как полином от $n$.

Пусть сначала $c=1$. Тогда по модулю тождеств $\mathbf{V}$ любой полилинейный полином от $x_{1}^{0}, \ldots, x_{m}^{0}, x_{1}^{1}, \ldots, x_{n-m}^{1}$ является линейной комбинацией левонормированных одночленов, начинаюшихся с произведения $x_{i}^{p} x_{j}^{q}, p, q$ равны 0 или 1 , а все остальные сомножители в них упорядочены по нижним индексам, причем сначала идут четные, а затем нечетные переменные. Количество таких одночленов растет не быстрее $n^{2}$, поэтому при $c=1$ теорема доказана. 
При $c>1$ воспользуемся индукцией по $c$. Это значит, что мы можем ограничиться подсчетом одночленов от $x_{1}^{0}, \ldots, x_{m}^{0}, x_{1}^{1}, \ldots, x_{n-m}^{1}$ вида $b_{1} b_{2} \ldots b_{c}$, где $b_{i}-$ левонормированное произведение переменных, в котором все четные переменные стоят в начале, и как четные, так и нечетные переменные упорядочены по возрастанию, начиная с третьей позиции. Перепишем тождество (20) как соотношение

$$
\begin{aligned}
& \left(z_{1} z_{2} x_{N+1} \ldots x_{2 N}\right) t_{1} \ldots t_{r}\left(z_{3} z_{4} x_{1} \ldots x_{N}\right) \\
& \quad \equiv \sum \alpha_{i} t_{0}\left(z_{1} z_{2} x_{i_{1}} \ldots x_{i_{N}}\right) t_{1} \ldots t_{r}\left(z_{3} z_{4} x_{j_{1}} \ldots x_{j_{N}}\right)
\end{aligned}
$$

где в правой части стоит линейная комбинация одночленов, у которых один из индексов $i_{1}, \ldots, i_{N}$ не больше $N$.

Поскольку $\mathbf{V} \subset \mathbf{N}_{c} \mathbf{A}$, то в свободной супералгебре $L$ многообразия $\mathbf{V}$ в силу (22) на однородных по градуировке переменных выполняется равенство

$$
\begin{array}{r}
b_{1} \ldots b_{i-1}\left(b_{i} x_{N+1} \ldots x_{2 N}\right) b_{i+1} \ldots b_{j-1}\left(b_{j} x_{1} \ldots x_{N}\right) \ldots b_{c} \\
\equiv \sum \alpha_{q} b_{1} \ldots\left(b_{i} x_{i_{1}} \ldots x_{i_{N}}\right) \ldots\left(b_{j} x_{j_{1}} \ldots x_{j_{N}}\right) \ldots b_{c}
\end{array}
$$

для любых $b_{1}, \ldots, b_{c} \in L^{2}$ с теми же условиями на $i_{1}, \ldots, i_{N}$, что и в $(22)$. Отсюда следует, что по модулю тождеств многообразия $\mathbf{V}$ любой полилинейный полином от $x_{1}^{0}, \ldots, x_{m}^{0}, x_{1}^{1}, \ldots, x_{n-m}^{1}$, лежащий в $\left(\mathscr{L}^{2}\right)^{c}$, выражается через произведения $a_{1} \ldots a_{c}$, где каждый $a_{i}$ - левонормированный одночлен вида

$$
a_{i}=b_{i} x_{i_{1}}^{1} \ldots x_{i_{s}}^{1},
$$

$b_{i}$ - одночлен степени не выше $m+2$, зависящий от некоторых из $x_{1}^{0}, \ldots, x_{m}^{0}$ и не более чем двух $x_{j}^{1}$. Последовательность $i_{1}, \ldots, i_{s}$ в $(24)$ упорядочена по возрастанию. Число произведений $a_{1} \ldots a_{c}$, у которых для всех одночленов $a_{i}$, кроме одного, выполняется условие $s \leqslant 2 N$, растет с ростом $n$ полиномиально.

Рассмотрим те одночлены $a_{1} \ldots a_{c}$, у которых

$$
a_{i}=b_{i} x_{i_{1}}^{1} \ldots x_{i_{s}}^{1}, \quad a_{j}=b_{j} x_{j_{1}}^{1} \ldots x_{j_{t}}^{1}, \quad s, t>2 N, \quad i<j .
$$

Из (23) следует, что можно ограничиться такими элементами, у которых $i_{s-N+1}<$ $j_{N}$. Покажем, что это гарантирует полиномиальный рост. Рассмотрим для наглядности случай, когда все $a_{1}, \ldots, a_{c}$ имеют большую степень. Тогда

$$
a_{1} \ldots a_{c}=\left(b_{1} x_{i_{1}}^{1} \ldots x_{i_{s}}^{1}\right)\left(b_{2} x_{j_{1}}^{1} \ldots x_{j_{t}}^{1}\right) \ldots\left(b_{c} x_{l_{1}}^{1} \ldots x_{l_{q}}^{1}\right)
$$

и $s, t, \ldots, q>2 N$. Строка индексов $i_{1}, \ldots, i_{s}, j_{1}, \ldots, l_{q}$ должна удовлетворять условию

$$
i_{1}<\cdots<i_{s-N+1}<j_{N}<\cdots<j_{t-N+1}<\cdots<l_{N}<\cdots<l_{q} .
$$

Количество строк из чисел $1, \ldots, n$, удовлетворяюших условию (25), не превьшает $t ! C_{n}^{t}$, где $t=2 N(c-1)$, т.е. растет полиномиально с ростом $n$. Отсюда следует полиномиальность роста многообразия $\mathbf{V}$, и теорема 1 доказана полностью.

Напомним, что $\mathbf{V}$ назьвают многообразием промежуточного роста, если асимптотически $\gamma_{n}(\mathbf{V})<a^{n}$ для любого $a>1$ и $\gamma_{n}(\mathbf{V})>n^{k}$ для любого целого $k$. Из теоремы 1 и полученных ранее оценок вытекает теорема 2. 
ДоКАЗАТЕЛЬСТво ТЕОРЕМЫ 2. Если рост $\mathbf{V}$ меньше, чем $2^{\frac{n}{2}-1}$, то $\mathbf{V} \subset \mathbf{N}_{c} \mathbf{A}$ по теореме 1 и лемме 2. Зафиксируем это число $c$. Если $\gamma_{n}(\mathbf{V})<2^{\frac{n}{4 c}}$, то по леммам 3 и 4 многообразие $\mathbf{V}$ удовлетворяет также условиям 2) и 3 ) теоремы 1 , которая гарантирует полиномиальность роста $\mathbf{V}$, и теорема 2 доказана.

Последнее утверждение аналогично результату в обычных алгебрах Ли, однако в лиевском случае можно указать нижнюю оценку: если $\gamma_{n}(\mathbf{V})<2^{n}$, то $\mathbf{V}$ - многообразие полиномиального роста [5]. Для супералгебр Ли вопрос о существовании подобной оценки открыт.

Напомним еше одно определение. Об многообразии $\mathbf{V}$ говорят, что оно почти полиномиального роста, если рост последовательности $\gamma_{n}(\mathbf{V})$ строго больше полиномиального, но у любого собственного подмногообразия рост полиномиален. Пример такого многообразия дает var $H$, где $H$ - четырехмерная супералгебра из леммы 2.

СлЕДСТВИЕ 1. $\operatorname{var} H$ - многообразие почти полиномиального роста.

ДоКАЗАТЕЛЬСТво. То, что рост многообразия $\mathbf{U}=\operatorname{var} H$ экспоненциален, доказано в лемме 3 . Пусть $\mathbf{V} \subset \mathbf{U}, \mathbf{U} \neq \mathbf{V}$. Тогда по лемме 1 в $\mathbf{V}$ выполняется тождество $(\operatorname{ad} y)^{n} \equiv 0$ для нечетной переменной $y$. Линеаризуя соотношение

$$
z_{1} z_{2} z_{3} \underbrace{y \ldots y}_{m} \equiv 0
$$

и учитывая (11), получаем тождество

$$
z_{1} z_{2} z_{3} y_{1} \ldots y_{m} \equiv 0
$$

для нечетных $y_{1}, \ldots, y_{m}$ и любых $z_{1}, z_{2}, z_{3}$. Отсюда и из (11) следует включение $\mathbf{V} \subset \mathbf{N}_{c} \mathbf{A}$ для достаточно большого $c$. Кроме того, из (11) и (26) следует, что любой одночлен, содержаший более $m+3$ нечетных сомножителей, равен нулю. Поскольку $\mathbf{U} \subset \mathbf{A} \mathbf{N}_{2}$, то в $\mathbf{V}$ выполняется и система тождеств (20) уже при $N=1$. Таким образом, все условия теоремы 2 выполнены, и следствие 1 доказано.

Наряду с известными примерами многообразий алгебр Ли почти полиномиального роста (см. [9]) и var $H$, этим свойством обладает и многообразие $\mathbf{A}^{2}$.

СЛЕДСТвИЕ 2. Многообразие всех метабелевых супералгебр Ли имеет почти полиномиальный рост.

ДоКАЗАТЕЛЬСТво. Поскольку произведение $x_{1} \ldots x_{m} y_{1} \ldots y_{m}$ не равно нулю в свободной метабелевой супералгебре Ли, где $x_{1}, \ldots, x_{m}$ - четные, а $y_{1}, \ldots, y_{m}-$ нечетные переменные, многообразие $\mathbf{A}^{2}$ не удовлетворяет условиям теоремы, поэтому его функция роста не полиномиальна. Если же $\mathbf{V}$ - собственное подмногообразие в $\mathbf{A}^{2}$, то $\mathbf{V}$ мультинильпотентно, т.е. сушествуют такие $m$ и $n$, что любое произведение $m$ четных и $n$ нечетных элементов равно нулю (см. [14, теорема 4$]$ ). Это означает вьполнение условия 2) из теоремы 2 . Поскольку 1) и 3 ) для $\mathbf{V} \subset \mathbf{A}^{2}$ очевидны, то рост $\mathbf{V}$ полиномиален, и следствие 2 доказано.

В заключение отметим, что для градуированных алгебр возможен и другой подход к определению функции роста. Если $L=L_{0} \oplus L_{1}=\operatorname{alg}\left\{x_{1}, x_{2}, \ldots, y_{1}, y_{2}, \ldots\right\}-$ свободная супералгебра счетного ранга некоторого многообразия $\mathbf{V}, x_{i} \in L_{0}$, 
$y_{i} \in L_{1}$, то можно рассмотреть пространство $P_{m, k}(L)$ полилинейных полиномов от $x_{1}, \ldots, x_{m}, y_{1}, \ldots, y_{k}$. Обозначим

$$
P_{n}^{\prime}(L)=\sum_{k=0}^{n} P_{k, n-k}(L)
$$

и положим

$$
\mu_{n}(\mathbf{V})=\operatorname{dim} P_{n}^{\prime}(L)=\sum_{k=0}^{n} \operatorname{dim} P_{k, n-k}(L) .
$$

Тогда последовательность $\left\{\mu_{n}(\mathbf{V})\right\}, n=1,2, \ldots$, также характеризует многообразие $\mathbf{V}$, и ее можно взять в качестве другой функции роста, отличной от $\gamma_{n}(\mathbf{V})$. Именно этот рост рассматривался в работе [14]. То, что эти функции различны, показывает пример метабелева многообразия, поскольку $\mu_{n}\left(\mathbf{A}^{2}\right)=n^{2}-1$ (см. [14]), а $\gamma_{n}\left(\mathbf{A}^{2}\right)$ растет экспоненциально. Аналогичная ситуация и с $\mathbf{U}=\operatorname{var} H$, поскольку $\gamma_{n}(\mathbf{U})>2^{\frac{n}{2}}$ по лемме 3, а $\mu_{n}(\mathbf{U}) \leqslant n^{2}$ в силу (11).

\section{Список литературы}

1. Regev A. Existence of identities in $A \otimes B / /$ Israel J. Math. 1972. V. 11. P. 131-152.

2. Воличенко И.Б. О многообразии алгебр Ли $A N_{2}$ над полем характеристики нуль // ДАН БССР. 1981. Т. 26. № 12. С. 1063-1066.

3. Петроградский В. М. О типах сверхэкспоненциального роста тождеств в РІ-алгебрах Ли // Фундаментальная и прикл. математика. 1995. Т. 1. №6. С. 989-1007.

4. Drensky V., Regev A. Exact asymptotic behaviour of the codimensions of some P.I. algebras // Israel J. Math. 1996. V. 96. P. 231-242.

5. Мищенко С. П. Нижние оценки размерностей неприводимых представлений симметрических групп и показателей экспоненты многообразий алгебр Ли // Матем. сб. 1996. T. 187. № 1. C. 83-94.

6. Кемер А.Р. Шпехтовость $T$-идеалов с полиномиальным ростом коразмерностей // Сиб. матем. журн. 1978. Т. 19. С. 54-69.

7. Бенедиктович И. И., Залесский А.Е. Т-идеалы свободной алгебры Ли с полиномиальным ростом последовательности коразмерностей // Вести АН БССР. 1981. №3. C. $5-10$.

8. Мищенко C. П. О многообразии полиномиального роста алгебр Ли над полем характеристики нуль // Матем. заметки. 1986. Т. 40. №6. С. 713-721.

9. Мищенко С. П. Рост многообразий алгебр Ли // УМН. 1990. Т. 45. №6. С. 25-45.

10. Scheunert M. The Theory of Lie Superalgebras. An Introduction // Lect. Notes Math. V. 716. Springer-Verlag, 1979.

11. Bahturin Yu. A., Mikhalev A.A., Petrogradsky V. M., Zaicev M. V. Infinite-dimensional Lie superalgebras. Berlin: Walter de Gruyter, 1992.

12. Зайчев M. В. Локально представимые многообразия супералгебр Ли // Матем. заметки. 1992. Т. 52. №5. С. 33-41.

13. Джеймс Г. Теория представлений симметрических групп. М.: Мир, 1982.

14. Бахтурин Ю.А., Дренски В.С. Тождества разрешимых цветных супералгебр Ли // Алгебра и логика. 1987. Т. 26. № 4. С. 403-418. 